Journal of Electronic Testing

June 2018, Volume 34, Issue 3, Pages 281-290

http://dx.doi.org/10.1007/s10836-018-5710-4

http://archimer.ifremer.fr/doc/00425/53678/

(C) Springer Science+Business Media, LLC, part of Springer Nature 2018

\title{
On-chip Generation of Sine-wave Summing Digital Signals: an Analytic Study Considering Implementation Constraints
}

\author{
David-Grignot Stephane ${ }^{1,{ }^{*}}$, Lamlih Achraf ${ }^{1,2}$, Belhaj Mohamed Moez ${ }^{1}$, Kerzérho Vincent ${ }^{1}$, \\ Azaïs Florence ${ }^{1}$, Soulier Fabien ${ }^{1}$, Freitas Philippe ${ }^{1}$, Rouyer Tristan ${ }^{2}$, Bonhommeau Sylvain ${ }^{3}$, \\ Bernard Serge ${ }^{1}$
}

${ }^{1}$ LIRMM, CNRS-University of MontpellierMontpellier Cedex 5,France

2 Ifremer,MARBECSète,France

${ }^{3}$ Ifremer,DOILe Port,France

* Corresponding author : Stephane David-Grignot, email address : Stephane.David-Grignot@lirmm.fr

\begin{abstract}
:
In the context of biosensor as much as Built-In-Self Test (BIST), on-chip sine-wave signal generation is a recurring research topic. Considering the implementation constraints, it implies a trade-off between the amount of resources and the signal quality. An attractive solution consists in combining several digital signals to build this analog sine-wave. The objective of this paper is to give an analytic study of various potential digital-based solutions. Thanks to this study, we prove that the technique consisting in setting the phase shifts and various amplitude values of the square-wave signals is the most efficient approach. This study allows the selection of the optimal square-wave signal parameters to cancel loworder harmonics of the generated signal. We proposed a solution for specification-oriented definition of the architecture
\end{abstract}

Keywords : Harmonic cancelation, Sine-wave generation, On-chip signal generation 


\section{INTRODUCTION}

The design of integrated sine-wave signal generator is a long-term research topic. Such designs have several applications from the Built-In-Self Test (BIST) [1] to impedance spectroscopy for biosensor application [2]. The main objective of designing an integrated sine-wave signal generator is to overcome the need for off-chip generation and drive the signal to the internal node. The constraints to design such an integrated generator are the required silicon area and the accuracy of the generated sine-wave signal. In order to minimize the generator size and its sensitivity to manufacturing process variations, it is relevant to use digital resources to generate the sine-wave signal.

An attractive solution consists in combining digital signals to generate the analog sine-wave [1] [2] [3] [4] [5] [6] [7]. This solution, illustrated in Figure 1, consists of a first digital block generating square-wave signals with various characteristics (amplitude and/or relative phase). Then, these signals are provided to another block that performs a clever summing, aiming at cancelling some harmonics on the output signal. As square-wave signals have harmonics which amplitudes decrease by $1 / k$ ( $k$ is the order of the harmonic). The basic principle of harmonic cancelling is to sum square-wave signals with different characteristics in order to sum the fundamental frequency bin while cancelling the harmonics. This process allows low-order harmonic cancellation. Then, a low-pass filter is used to remove residual high-frequency harmonics.

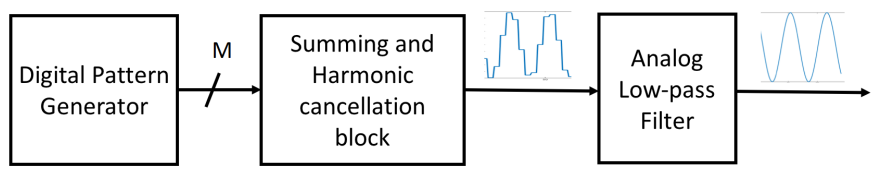

Fig. 1. Block diagram of low-distortion sine-wave generation using harmonic cancellation

In order to implement such a solution, the number of required signals, amplitudes, frequencies and relative phaseshifts have to be defined. The architectures described in the literature are strongly application-driven solutions. As a consequence, there isn't any theoretical analysis of the optimized values of the required square-wave signal parameters.

This paper is an extension of [8] where an analytic study of square-wave parameters for efficient sine-wave generation is performed and focuses on performances in terms of linearity of the generated sine-wave. This extension arbitrates on one sine wave generation technique in-light of the novel presented approach to mitigate mismatch issues.

In the second section, we define the parameters of the square-wave signals to be tuned in order to perform the harmonic cancellation. The third section provides the analytic study of harmonic cancellation for two different approaches. A solution to a mismatch issue during implementation is suggested in the fourth section. Finally, a study of the robustness of the best solution is provided in the fifth section.

\section{BASIC PRINCIPLE AND CONSTRAINTS}

\section{A. Square-wave signal parameters}

For changing the distortion of a generated sine-wave signal, several square-wave signal parameters can be tuned. For each square-wave signal $m$, these parameters are the amplitude $A_{m}$, the phase $\varphi_{m}$ and the duty cycle $r_{m}$. In order to optimize the harmonic cancellation with $M$ square-wave signals $3 \times M$ parameters have to defined. It will be shown in the following 
that it is possible to reduce this number of parameters that needs to be considered.

\section{B. Symmetry of generated square-wave signals}

As illustrated in figure 2, using $M$ square-wave signals to generate the sine-wave signal, if $M$ is an even number, $M / 2$ signals have symmetrical phase shift compare to the other $M / 2$ signals. In case of odd number of square-wave signals, $m=0$ signal is in-phase with the targeted sine-wave signal. Then $p=(M-1) / 2$ signals have symmetrical phase shift compared with the other $p$ signals. Then, we define the squarewave signal names $m$ going from $-p$ to $p$. Since the signals are symmetrical $A_{m}=A_{-m}$ and $\varphi_{m}=-\varphi_{-m}$.

The duty cycle can be set aside. Indeed, two square-wave signals with a $\varphi_{m}$ symmetrical phase shift are two signals with a duty cycle of $r_{m}=\frac{\pi-2 \varphi_{m}}{2}$. As a consequence any duty cycle can be generated with symmetrical signals.

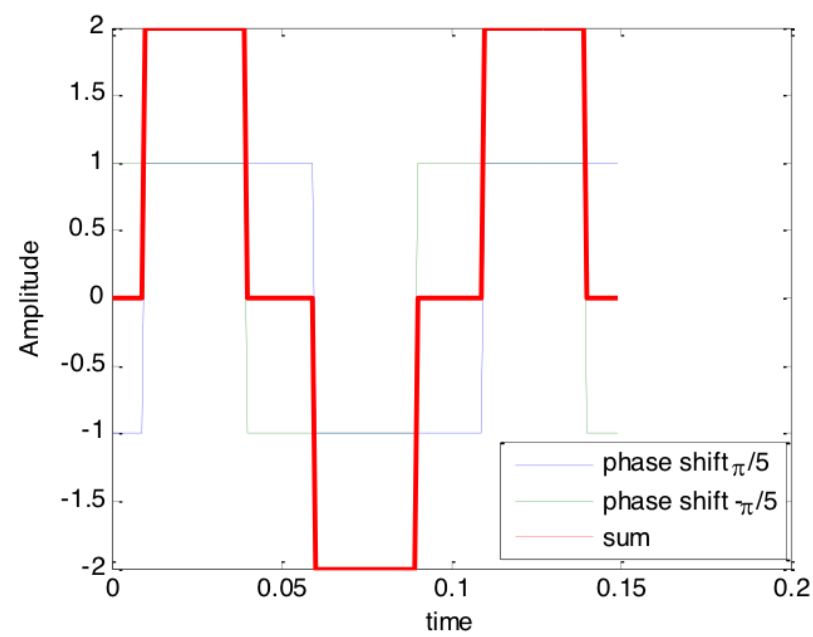

Fig. 2. Symmetry of sine-wave signal generated by summing square-wave signals

\section{Constraints and objective}

The objective of this paper is to evaluate the trade-off between 3 constraints:

- Quality of generated signal

- Implementation effort

- Robustness of the solution

Quality and robustness of generator architecture depends on hardware implementation limitations and constraints. For instance, it is easier to generate a phase shift as a fraction of the sine-wave period rather than an arbitrary phase-shift. In addition it is easier to generate signals with identical amplitudes rather than signals with arbitrary amplitudes.

Architecture implementation impacts on accuracy and robustness, but the linearity of the generated signal is also strongly related to the chosen parameters of the digital based generator.

\section{HARMONIC CANCELLATION}

Several options can be considered to define parameters of the digital-based generator. Each option has to be considered according to our objective and constraints (Quality, Implementation effort and Robustness).

- Is it efficient to strongly constrain the phase-shift of the square-wave signals and precisely adjust their amplitude?

- Is it more efficient to keep one amplitude for any square-wave signal and adjust precisely their relative phase-shift?

- Is adjusting precisely their phase-shift and amplitude the ultimate solution?

These different case studies will be discussed in the following sections.

\section{A. Fixed phase-shifts and adjustable amplitudes of square-} waves

A possible solution for harmonic cancellation is to set the phase-shifts $\varphi_{m}$ as ratios of the sine-wave period (keeping in mind implementation constraints) and adjust the amplitudes $A_{m}$ arbitrarily of each square signal to sum. Such a solution has been described in [7]. Values of phase-shifts $\varphi_{m}$ and amplitudes $A_{m}$ of each square-wave to sum are expressed in Equation 1.

$$
\left\{\begin{array}{l}
\varphi_{m}=2 \pi \frac{m}{N} \\
A_{m}=\cos \left(2 \pi \frac{m}{N}\right)
\end{array}\right.
$$

with $N=4(p+1)$ and $m=\llbracket-p, p \rrbracket$

We can show that this solution cancels the first harmonics analytically. Equation 2 gives the Fourier series of the sum of the $M$ square-wave signals.

$$
y(t)=\sum_{m=-p}^{p} \sum_{k=-\infty}^{\infty} C_{k, m} e^{i k \omega t}
$$

The Fourier coefficients given by equation 3 are the sum of the Fourier coefficients of the square-wave signals.

$$
C_{k}(y)=\sum_{m=-p}^{p} \frac{\cos \left(2 \pi \frac{m}{N}\right)}{k \pi}\left(1-e^{i k \pi}\right) e^{-i k m 2 \frac{2 \pi}{N}}
$$

Equation 4 is the development of equation 3.

$$
\begin{aligned}
C_{k}(y) & =\frac{2}{k \pi} \sum_{m=-p}^{p} \frac{e^{2 \pi \frac{m}{N}}+e^{-2 \pi \frac{m}{N}}}{2} e^{-i k m 2 \frac{2 \pi}{N}} \\
& =\frac{1}{k \pi} \sum_{m=-p}^{p} e^{2 \pi \frac{m}{N}(k-1)}+e^{-2 \pi \frac{m}{N}(k+1)}
\end{aligned}
$$


Considering that the sum of the roots of unity is zero. We can observe that $\sum_{m=-p}^{p} e^{-2 \pi \frac{m}{N}(k+1)}$ cancels when $k$ is not equal to $N-1$ (where, as defined previously, $N=4 p+1)$. This implies that $C_{k}(y)$ is equal to zero except for $k=\{1 ; 4(p+$ 1) $-1 ; 4(p+1)+1 ; 8(p+1)-1 ; 8(p+1)+1 ; \ldots\}$.

Thanks to this approach harmonics below $4(p+1)-1$ are cancelled. Thus, $M$ signals can cancel harmonics with order below $2 M+1$ for odd $\mathrm{M}$.

Figures 3 and 4 provide respectively the temporal and spectral representation of a sine-wave signal for five squarewave signals $M=5$.

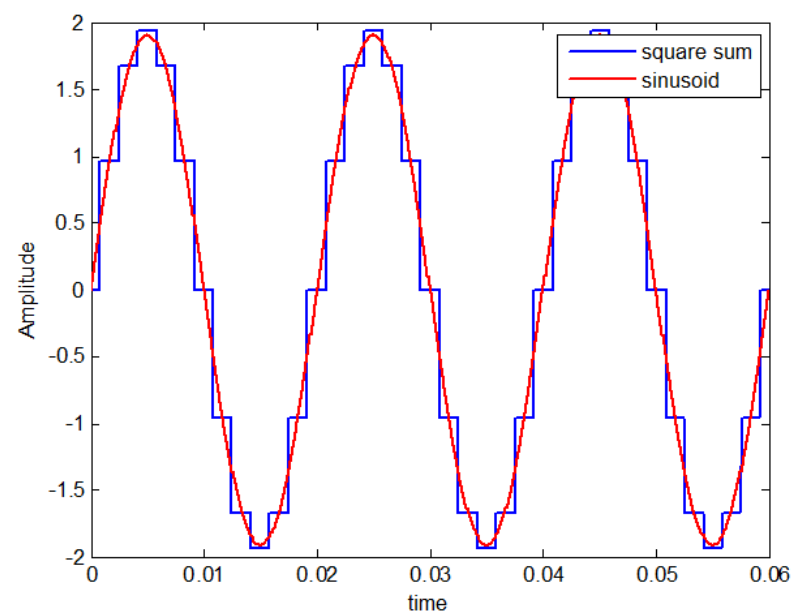

Fig. 3. Sine-wave signal generated using square-wave signals with various amplitudes for five signals $M=5$

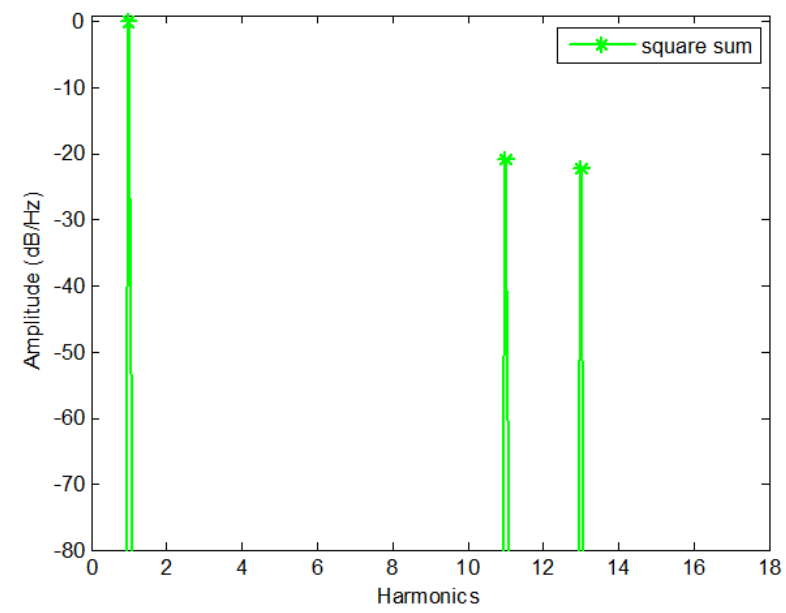

Fig. 4. Spectrum of sine-wave signal generated using square-wave signals with various amplitudes for five signals $M=5$

B. Fixed amplitudes and adjustable phase-shifts of squarewaves

The other option consists in setting the amplitudes to the same value and adjusting the phase-shifts to cancel the harmonics.

$$
\left\{\begin{array}{l}
\varphi_{m} \text { adjustable } \\
A_{m}=1
\end{array}\right.
$$

Two approaches can be used:

- A minimization technique, with a search over the phase shifts values for a minimum in the total harmonic distortion (THD).

- An analytic study with a theoretical approach.

\section{1) Minimization technique:}

Let us consider the Fourier coefficients of the generated signal:

$$
C_{k}(y)=\sum_{m=-p}^{p} \frac{-i}{k \pi} e^{-i k \varphi_{m}}, \text { for odd } \mathrm{k}
$$

To define the values of the $\varphi_{m}$ phase-shifts, we propose to minimize the harmonic distortion ratio provided by Equation 7

$$
T H D_{N_{h a r}}=\frac{\sqrt{\sum_{m=2}^{N_{h a r}}\left|C_{k}\right|^{2}}}{\left|C_{1}\right|}
$$

$N_{\text {har }}$ is the number of harmonics to be cancelled. For the first integer values of $N_{h a r}$ we run a search for a minimum on $T H D_{N_{h a r}}$ with equation 7 . We observe that it is possible to cancel TH $D_{N_{h a r}}$ when $N_{\text {har }}$ is below $M+3$ when the number of signals $M$ is even.

Figures 5 and 6 provide respectively the temporal and spectral representation of a sine-wave signal generated for six square-wave signals $M=6$

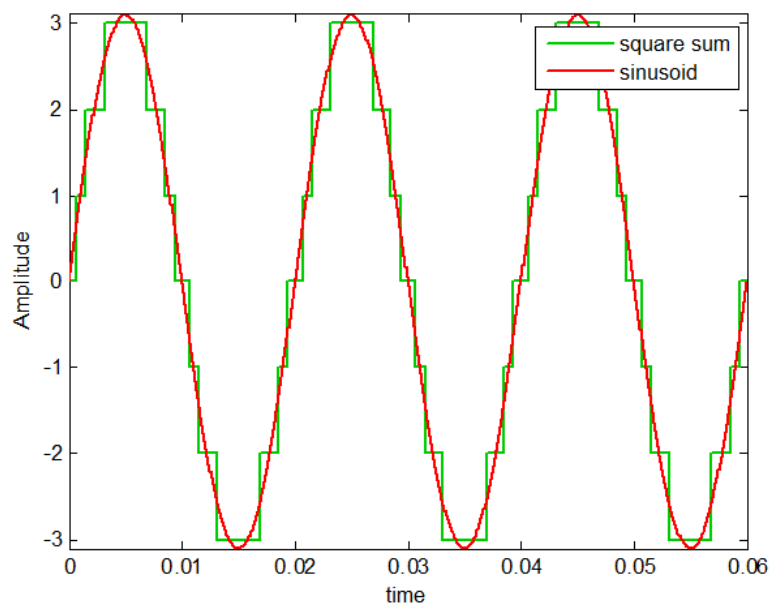

Fig. 5. Sine-wave signal generated using square-wave signals with various phase shifts for six signals $M=6$ with $\left[\varphi_{1} ; \varphi_{2} ; \varphi_{3}\right]=$ [0.2037; 0.4701; 0.9784] rads 


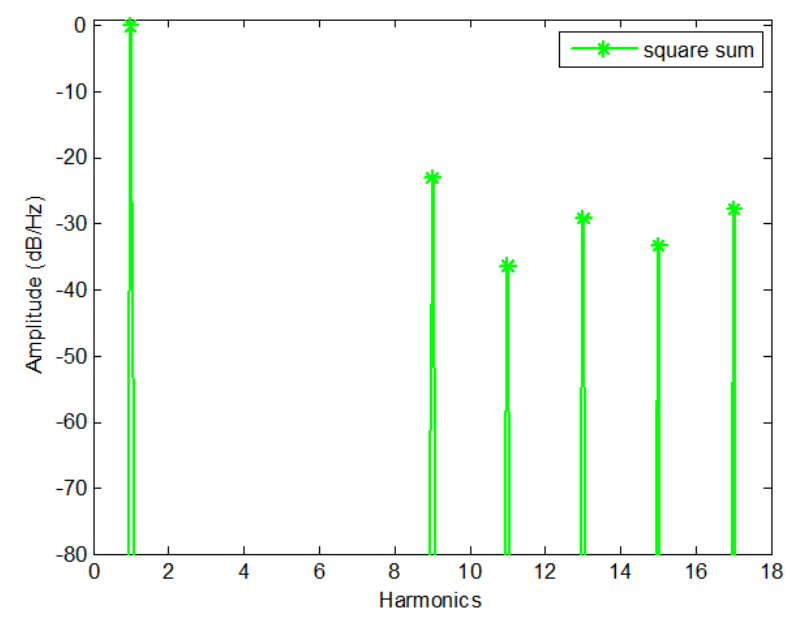

Fig. 6. Spectrum of sine-wave signal generated using square-wave signals with various phase shifts for six signals $M=6$

The approach proposed in chapter III.A (Fixed phase-shifts and adjustable amplitudes of square-waves) can already be considered more efficient because it cancels more harmonics with less required square-wave signals. In addition, it seems that the requested accuracy of the phase-shift is very constraining. Let's consider the equation 8 that provides the Fourier series coefficient affected by a phase-shift error.

$$
C_{k}(y)=\sum_{m=-p}^{p} \frac{-i}{k \pi} e^{-i k\left(\varphi_{m}+2 \pi \text { deviation }\right)}, \text { for } \mathrm{k} \text { odd }
$$

Figure 7 presents the harmonic distortion ratio variation for a phase-shift deviation from $10^{-4}$ to $10^{-2}$ of a period.

As presented by Figure 7, we observe by simulation that harmonic distortions affected by a phase-shift error of $10^{-3}$ of a period, have an amplitude of $-50 \mathrm{~dB}$.

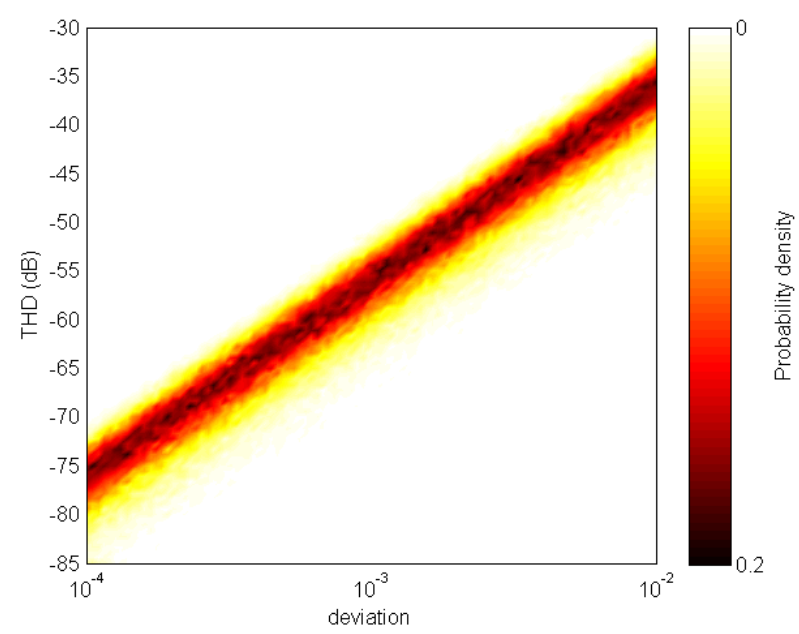

Fig. 7. Harmonic distortion ratio variations due to varying phase-shift error Monte-Carlo results for a sine-wave signal generated using square-wave signals with various phase shifts for six signals $M=6$
To give an example, as illustrated by figure 7 , in order to reach a harmonic cancellation of the order of $-50 \mathrm{~dB}$ when generating a sine-wave of frequency $10 \mathrm{MHz}$, a digital frequency higher than $10 \mathrm{GHz}$ is necessary. This number implied a very high clock frequency generator involving high constraint.

\section{2) Analytic approach:}

[9] has developed analytically some solutions that cancel the harmonics by summing square signals with unitary amplitudes. The values of phase shifts are therefore analytically known and represent a reasonable fraction of the period of the main signal to generate.

The analytic solutions, written as vectors, are :

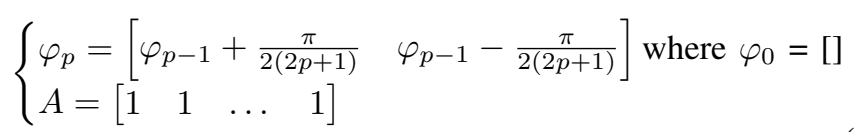

$\mathrm{p}$ is the number of odd harmonics cancelled. For example to cancel harmonic 3 and 5

$$
\left\{\begin{array}{l}
\varphi_{2}=\left[\begin{array}{llll}
\pi / 6+\pi / 10 & -\pi / 6+\pi / 10 & \pi / 6-\pi / 10 & -\pi / 6-\pi / 10
\end{array}\right] \\
A=\left[\begin{array}{llll}
1 & 1 & 1 & 1
\end{array}\right]
\end{array}\right.
$$

This method permits to have phase shifts that represent an integer division of the period of the signal generated. This permits a much simpler implementation as it is very difficult to generate arbitrary phase shifts like it is requested in chapter III.B.1). However the number of square signals that have to be summed grows exponentially with the number of harmonics to cancel.

\section{Adjustable amplitudes and adjustable phase-shifts of square-waves}

Releasing the constraints on amplitudes and phase shifts doesn't seem to enable the cancellation of more harmonics. Without an analytic theory it is impossible to conclude with certainty on this fact. However, we have sought to minimize the next harmonic with the minimization of $T H D_{N_{h a r}}$ method in simulation and have not found any improvement. Moreover, an intuitive analysis of the number of parameters and the number of harmonics to cancel leads us to think there is no improvement possible. Indeed, taking five signals $M=5$ as example, since amplitudes and phase shifts are relative to other square waves added, the parameters permitted are the amplitude ratio and the phase shift of 4 signals, adding up to 8 parameters. Since the waveform is even, the parameters are symmetric two by two, leading to only 4 parameters adjustable. The adjustable amplitudes method already permits to cancel the first 4 odd harmonics : 3, 5, 7 and 9. This does not provide any more degrees of freedom for parameters to cancel another harmonic. The default phase shifts used in the adjustable amplitude method seem already optimum.

We conclude that in fact adding adjustable phase shifts to the adjustable amplitude method does not provide any improvement in the number of harmonics cancelled. 


\section{Conclusion}

As mentioned in section II-A one of the parameters to vary is $M$, the number of square-wave signals to generate. $M$ can then be linked to the number of harmonics to cancel. The number of square-wave generators to design will be thus linked to the specification of the targeted application.

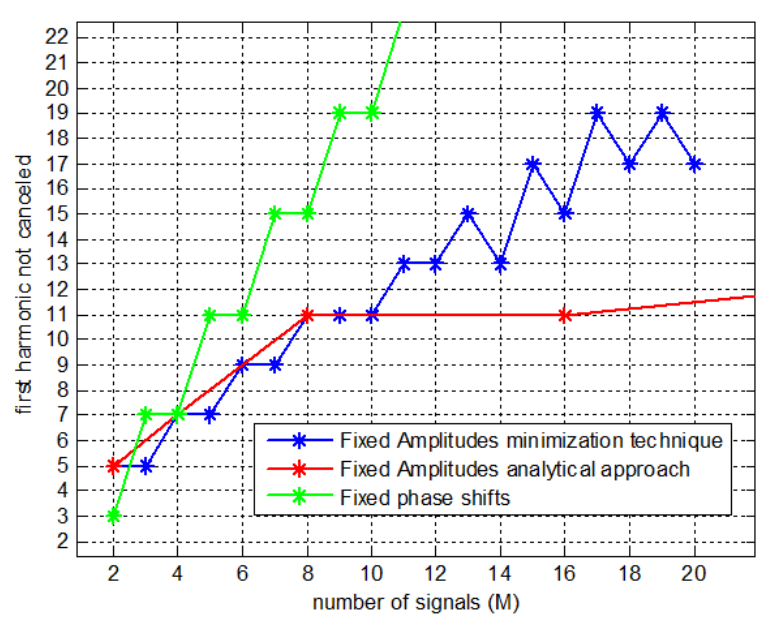

Fig. 8. Comparison of harmonic cancellation techniques

Figure 8 shows the performances of the various solutions to cancel harmonics. Each one has pros and cons.

The fixed amplitudes with minimization technique has the benefit of having equal amplitudes of square-wave signals but the phase shift are not known with precision because they are not determined analytically.

Fixing amplitudes and determining analytically the phase shifts as described in [9] offers a reasonable division of the main period. This allows a suitable implementation of the clock generation. Theoretically, this technique only cancels harmonics in a logarithmic fashion with the number of signals but still stays as efficient as the minimization technique for 8 or less signals.

Among these techniques, the technique using real coefficients as ratios of amplitudes for the summed squarewave allows to cancel the most harmonics. Moreover, the phase shifts generated are the largest and thus the easiest to implement. However, since the ratios are irrational numbers this technique is hard to implement for matching purposes.

\section{IMPLEMENTATION}

We seek to benefit from the technique that cancels the most harmonics (i.e. Fixed phase shifts described in section III.A.) without matching issues.

The generation of square-wave forms with ratio amplitudes can be implemented through resistor voltage divider or current mirrors.

In order to facilitate the matching of the devices (resistors or transistors) in the implementation, it is required that the ratios between the amplitudes of the signals to be added are integer fractions. As illustrated in Figure 13, introducing approximated ratios leads to harmonic distortions. Figure 9 has been computed in the particular case as an example of:

$$
\begin{gathered}
\left\{\begin{array}{l}
\varphi_{m}=\frac{\pi}{6}, \frac{\pi}{3} \\
A_{m}=\frac{1}{2}, \frac{\sqrt{(3)}}{2}
\end{array}\right. \\
\text { approximated to }\left\{\begin{array}{l}
\varphi_{m}=\frac{\pi}{6}, \frac{\pi}{3} \\
A_{m}=\frac{1}{2}, \operatorname{round}\left(\frac{\sqrt{(3)}}{2} d\right) \frac{1}{d}
\end{array}\right.
\end{gathered}
$$

We thus seek the integer fraction that introduces the lowest error. We observe in Figure 9 that it is not necessarily the integer fraction with the largest denominator that introduces the lowest distortion.

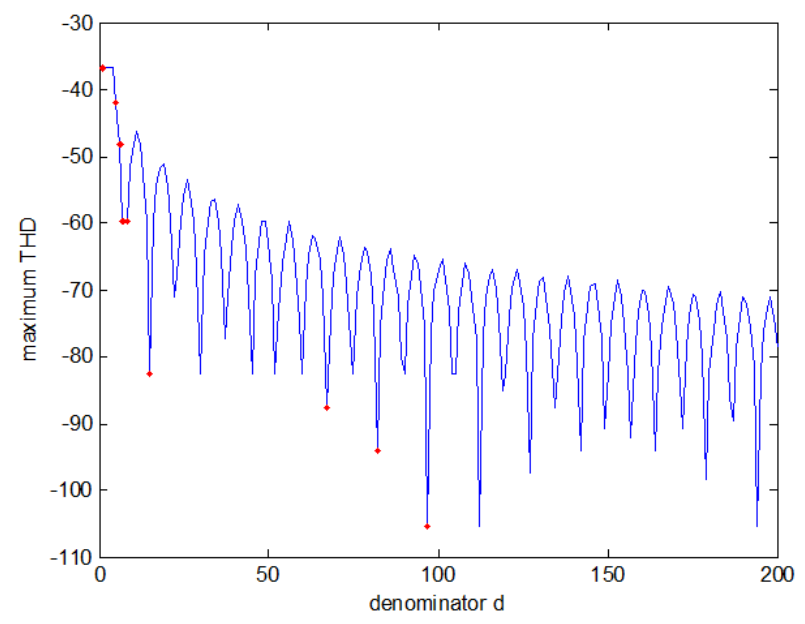

Fig. 9. Harmonic distortion of a sine-wave generated using square-wave signals with approximated amplitudes with fraction with denominator $d$

A better approach to approximate a real number with a rational number is through continued fractions of the form described in Equation 13. Indeed, if we take the continued fraction expansion of an irrational number $\alpha$ and cut it off after $n$ iterations, this resulting rational number is known as the $n^{\text {th }}$ convergent of $\alpha$. From The Law of Best Approximates, a theorem by Lagrange (Theorem 5.9 in [10]), these convergents are precisely the best approximates of an irrational number. Theses values are reported as red dots in Figure 9.

$$
a=a_{0}+\frac{1}{a_{1}+\frac{1}{a_{2}+\frac{1}{a_{3}+\ddots} .}}(13)
$$

One can compute analytically the harmonic distortions generated with the signal composed of square-waves of amplitudes with the approximation up to $n$ iterations on the continued fractions. 


$$
A_{m n}=a_{m 0}+\frac{1}{a_{m 1}+\frac{1}{a_{m 2}+\ldots+\frac{1}{a_{m n}}}}
$$

The more iterations we proceed on the continued fraction, the more precise it becomes as illustrated in Figure 10 .

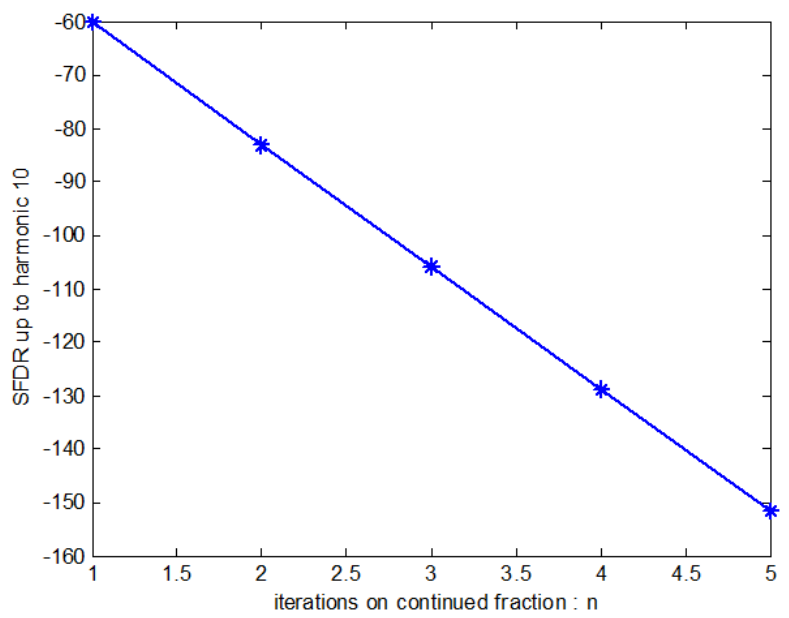

Fig. 10. Spurious Dynamic Range (SFDR) of a sine-wave generated using square-wave signals with approximated amplitudes with iteration on continued fraction

We can plot the THD in Figure 11 and SFDR in Figure 12, where $N_{\text {Har }}=11$ in equation 7 , and SFDR up to a given harmonic in function of the number of signals used and the number of iterations on the continued fraction. We can see that, although the SFDR is linear with the number of iterations on continued fraction (as shown in Figure 10), for any given number of signals it varies from one number of square signals to another.

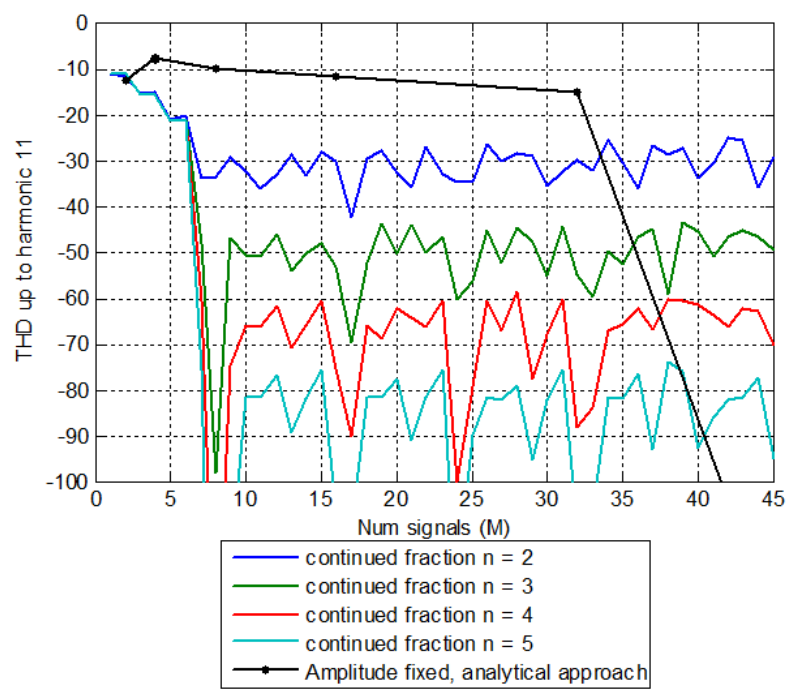

Fig. 11. Total harmonic distortion (THD) up to harmonic 11 of a sine-wave generated using square-wave signals with approximated amplitudes with $n$ iterations on continued fractions

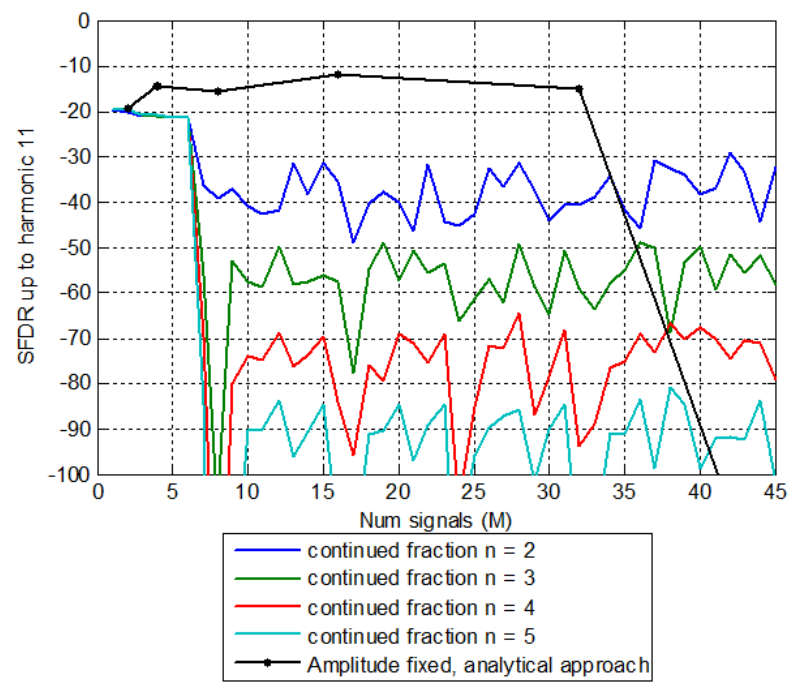

Fig. 12. Spurious Dynamic Range (SFDR) of a sine-wave generated using square-wave signals with approximated amplitudes with $\mathrm{n}$ iterations on continued fractions

However, the number of iterations on continued fraction does not necessarily represent the complexity of the current mirror, or resistance bridge, that have to be implemented. Indeed, the number of devices needed would rather be proportional to the sum of the denominator and the numerator. It is thus up to the designer to consider a solution with less square signals to sum but lots of devices as suggested in this paper or a solution with more signals to sum but a less complex design on each of these and a faster clock generation as suggested in [9]. 


\section{ROBUSTNESS STUDY}

Even if robustness is implicitly high because of the digitalbased approach of the proposed methods, the impact of errors on the linearity for the generation were studied using adjustable amplitudes of square-wave signals. For this purpose, an example was used with amplitudes defined as follows :

$$
\begin{gathered}
\left\{\begin{array}{l}
\varphi_{m}=\frac{\pi}{6}, \frac{\pi}{3} \\
A_{m}=\frac{1}{2}, \frac{\sqrt{(3)}}{2}
\end{array}\right. \\
\text { approximated to }\left\{\begin{array}{l}
\varphi_{m}=\frac{\pi}{6}, \frac{\pi}{3} \\
A_{m}=\frac{1}{2}, \frac{13}{15}
\end{array}\right.
\end{gathered}
$$

In order to approximate $\frac{\sqrt{3}}{2}$, we use the algorithm presented in the previous section, with continued fraction expansion up to the 3rd iteration, as it is a good compromise between precision and design complexity. Possible variations are phaseshift error, jitter and gain error on amplitude. Jitter is a random value normally distributed added to the time variable in the simulation.

Figure 13 presents the spectrum of the sine-wave generated with $p=2$, this is five square signals summed up : $M=5$. This sine-wave signal is affected by jitter and/or gain error of the amplitude of the square-wave signals. We observe that the error on the amplitude of the square-wave induces an amplitude increase of the harmonics initially cancelled. The jitter induces an increase of the noise level. The study in [11] presents a sinusoidal signal synthesizer architecture based on a fixed phase shifts with a similar approach. A robustness study has also been conducted by sweeping the amplitude and phase errors using transistor level monte-carlo simulation data. It is interesting to note that the HD3 transistor-level simulation results are similar to the harmonic distortion results of our analytic model.

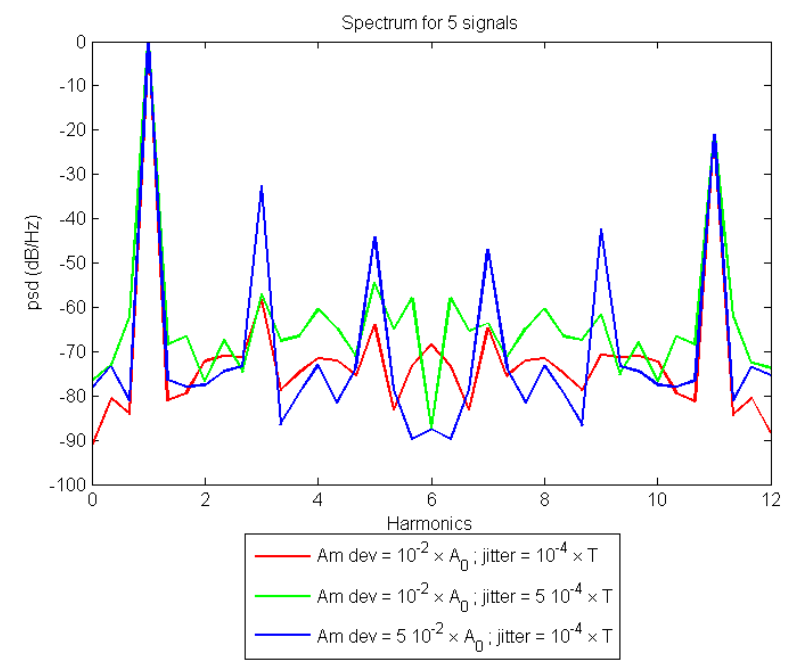

Fig. 13. Spectrum of a sine-wave signal affected by amplitude error and/or jitter on the square-wave signals for sine-wave signal generated using squarewave signals with various amplitudes five signals $M=5$
Figure 14 presents the computation of the harmonic distortion for various values of jitter. The jitter has also an impact that is significant on the harmonic distortion ratio. When the jitter is large, the noise floor created dominates the distortions due the amplitude deviation.

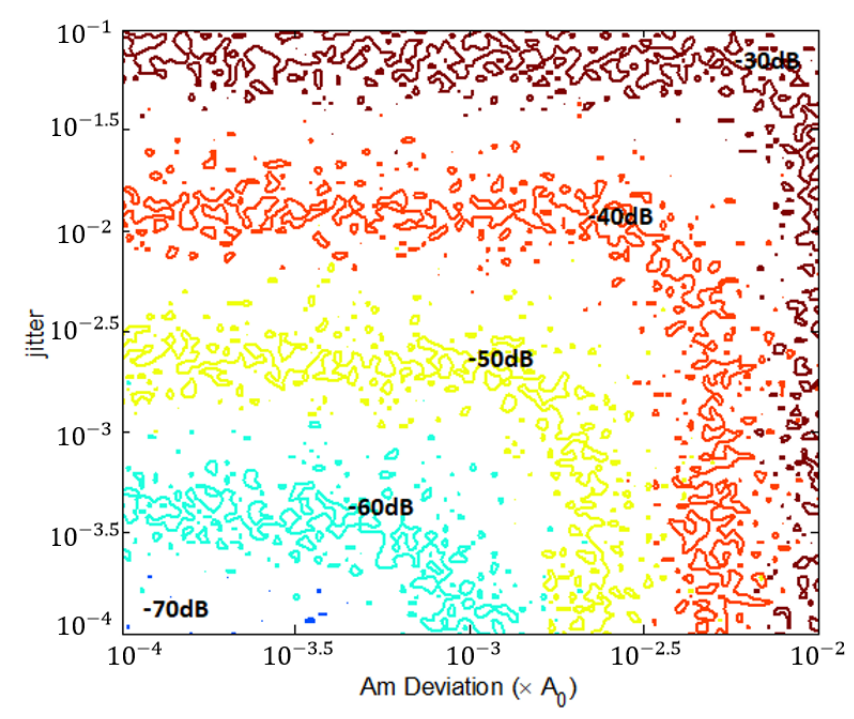

Fig. 14. Harmonic distortion ratio of a sine-wave generated using squarewave signals with various amplitudes deviations and affected by various level of jitter

According to the analytic study and simulation results we propose some features for an efficient implementation. Using five sine-wave signals, we can cancel harmonics below the eleven order. To generate $10 \mathrm{MHz}$ sine-wave signal, a 120 $\mathrm{MHz}$ clock frequency is needed. The architecture with fixed phase shift and variable amplitudes is optimally demanding for its digital clock as it is a multiple of the generated frequency. In order to have harmonic distortions at a maximum of $-50 \mathrm{~dB}$, jitter should be below $0.1 \mathrm{~ns}$ and the amplitude error should be limited to $1 \%$. This approach can be used in any other combination of frequency, amplitudes and precision needed.

\section{CONCLUSION}

This work is in the field of generating sine-wave signals by summing square-wave signals in order to cancel low-order harmonics. The effectiveness of the harmonic cancellation is related to the characteristics of the square-wave signals. Two approaches are generally implemented. The first approach consists in setting the phase shifts according to the implementation technique and setting various amplitude values of the squarewave signals. The second approach is the inverse. It consists in setting the different amplitudes at the same value and setting various phase shifts of the square-wave signals. We have analytically proven that for a given number of square-wave generators, the first approach allows to cancel a higher number of harmonics. In addition, considering targeted specifications, we have proven by simulation that the first approach is less constraining in terms of clock generation. We have also offered a solution to the matching issue due to the irrational nature of the ratios. Thanks to a continued fraction development of the ratios computed analytically, a satisfying sine-wave approximation can be generated. 


\section{ACKNOWLEDGMENT}

This research was part of the MERLIN-POPSTAR project funded by Ifremer and a $\mathrm{PhD}$ grant funded by Ifremer and labex Numev. We thank our colleagues from Ifremer/REM/RDT and RBE, CNRS, UM and IRD who provided insight and expertise that greatly assisted the research.

\section{REFERENCES}

[1] M. J. Barragan, G. Leger, D. Vazquez, and A. Rueda, "On-chip sinusoidal signal generation with harmonic cancelation for analog and mixed-signal bist applications," Analog Integrated Circuits and Signal Processing, vol. 82, no. 1, pp. 67-79, 2015. [Online]. Available: http://dx.doi.org/10.1007/s10470-014-0456-0

[2] D. Rairigh, X. Liu, C. Yang, and A. J. Mason, "Sinusoid signal generator for on-chip impedance spectroscopy," in 2009 IEEE International Symposium on Circuits and Systems, May 2009, pp. 1961-1964.

[3] M. M. Elsayed and E. Sanchez-Sinencio, "A low thd, low power, high output-swing time-mode-based tunable oscillator via digital harmoniccancellation technique," IEEE Journal of Solid-State Circuits, vol. 45, no. 5, pp. 1061-1071, May 2010.

[4] B. K. Vasan, S. K. Sudani, D. J. Chen, and R. L. Geiger, "Low-distortion sine wave generation using a novel harmonic cancellation technique," IEEE Transactions on Circuits and Systems I: Regular Papers, vol. 60, no. 5, pp. 1122-1134, May 2013.

[5] P. Aluthwala, N. Weste, A. Adams, T. Lehmann, and S. Parameswaran, "A simple digital architecture for a harmonic-cancelling sine-wave synthesizer," in 2014 IEEE International Symposium on Circuits and Systems (ISCAS), June 2014, pp. 2113-2116.

[6] C. Shi and E. Snchez-Sinencio, "150-850 mhz high-linearity sinewave synthesizer architecture based on fir filter approach and sfdr optimization," IEEE Transactions on Circuits and Systems I: Regular Papers, vol. 62, no. 9, pp. 2227-2237, Sept 2015.

[7] H. Malloug, M. J. Barragan, S. Mir, E. Simeu, and H. Le-Gall, "Mostly-digital design of sinusoidal signal generators for mixed-signal bist applications using harmonic cancellation," in 2016 IEEE 21st International Mixed-Signal Testing Workshop (IMSTW), July 2016, pp. $1-6$.

[8] S. David-Grignot, A. Lamlih, V. Kerzerho, F. Azais, F. Soulier, S. Bernard, T. Rouyer, and S. Bonhommeau, "Analytical study of onchip generations of analog sine-wave based on combined digital signals," in 2017 IEEE 22nd International Mixed-Signal Testing Workshop (IMSTW), July 2017.

[9] H. Malloug, M. J. Barragan, S. Mir, and H. Le-Gall, "Harmonic cancellation strategies for on-chip sinusoidal signal generation using digital resources," in 2017 IEEE 22nd International Mixed-Signal Testing Workshop (IMSTW), July 2017.

[10] E. Burger, Exploring the Number Jungle: A Journey into Diophantine Analysis, 2000.

[11] C. Shi and E. Sanchez-Sinencio, "On-chip two-tone synthesizer based on a mixing-fir architecture," IEEE Journal of Solid-State Circuits, 2017. 